\title{
An Extensive Review on Recent Emerging Applications of Artificial Intelligence
}

\author{
B. Harini1), N.Thirupathi Rao')
}

\begin{abstract}
In recent days, the applications of artificial intelligence are growing in number by day to day. In the present paper, the authors gave the brief idea about artificial intelligence and the various applications of artificial intelligence. The utilization of artificial intelligence and its related technologies had encouraged and providing various facilities for researchers and scientists to develop and implement the artificial intelligence techniques in various emerging sectors of the market today. As the technology is growing day to day, the utilization of various applications by using the technology is also growing. Hence, the authors gave a clear idea and discussed in detail about the applications of artificial intelligence with the help of some diagrams.
\end{abstract}

Keywords : Artificial Intelligence, Applications of AI, Machine Learning, Robotics, Astronomy, Construction.

\section{Introduction}

In recent days, the big breakthrough or the revolution in the new technologies was the utilization of artificial intelligence in almost all devices or the applications that were being used by humans in the society. The list of these applications is increasing from day to today. The utilization was increasing from day to day in such a way that the number was not limiting and it is increasing from time to time. The utilization of smart technologies by the common people was increasing in a gradual way to high level. The current trend is not that much familiar when it is working with the similar sort of technologies in previous attempts and previous utilization. The number of applications that were following to the common people was increased a lot such that all the companies are trying to increase the business further more. As these companies are trying to increase the business, the common people reach to the smart technologies and smart devices utilization was increasing a lot[1]. The business and the

Received(April 12, 2019), Review Result(1st: April 30, 2019, 2nd: June 5, 2019), Accepted(June 10, 2019)

1) (Professor) Department of Computer Science and Engineering, Vignan's Institute of Information Technology (A), Visakhapatnam-530049, AP, India

2) (Professor, Corresponding Author) Department of Computer Science and Engineering, Vignan's Institute of Information Technology (A), Visakhapatnam-530049, AP, India

email: nakkathiru@gmail.com 
income of these companies are increasing in a high rate with these sorts of applications.

The changes in the utilization of this technology had brought so many changes in recent technologies. Such changes can be observed by using various devices for fast working and processing, smart technologies for smart processing and other devices for reducing the size of such devices had made the technologies more familiar and more famous. This technology had increased the machines to supply the intelligence of their own and to think of their own and process the tasks assigned to such machines for processing with their own thinking capacities. By preparing the database or the knowledge for the machines, the machines will decide based on the data available for such machine to take the decisions. The decisions will be purely based on the decisions that we are going to provide for the previous problems with a set of solutions. The success of the current machines will depend on the performance of the machine with the existing database or the knowledge base[2]. The database can be called here as the knowledge base.

Now days the machines are using and performing some advanced features or the advanced mode working with the intelligence sort of part for problem solving. The major changes or the major technological change that was being implemented was the utilization of cognitive intelligence rather than the natural intelligence. This change of technology or the utilization of these technology changes can be observe by the common public to the senior people with the help of the applications and devices that are available in the market today. The implementation and utilization of artificial intelligence had opened the ways and the routes for its utilization in various emerging sectors in the current society.

Some of these technologies are like the self driving cars, chatbots, sharing the data and chatting with other users, robotics, data recognition, pattern recognition, face detection and image processing applications. The utilization of artificial intelligence in these areas was creating a good and significant impact on the users and the senior researchers who were working in these areas. The utilization was increasing from day to day and the implementation of these technologies in current applications will increase and they will become more part of all our day to day lives. Various sectors of the society are increasing in the commendable areas where more income and more service to the society and more employment will be generated. Some of the various sectors in the society which were growing rapidly in these days are the financial advising and financial services to customers and citizens of the society, automotives and automatic self driving vehicles for reducing accidents and more security to the drivers and other important areas like the healthcare[3].

Some of the applications that are growing very fast and having the growth very rapidly in 
the current trends of technology can be observed in the following sections. Various set of applications where you can find the utilization of artificial intelligence in various applications can be observed and represented in the following sections in detail[4]. Those applications include,

1. Astronomy

2. Construction

3. Agriculture

4. Sports

5. Entertainment

6. Medical and Healthcare

7. Education

\section{Applications of Artificial Intelligence}

Some of the applications of artificial intelligence are discussed in detail in the current section as follows,

\subsection{Astronomy}

The most interesting and important subject for every human being is knowing the facts about the universe. Today, Science is advanced to such a level that scientists can be able to answer almost all questions related to the doubts of normal human beings. But, not every question of human beings was finding the answers. The subject related to such sort of things and other related questions was astronomy. The utilization of artificial intelligence in the current area would be more helpful for analyzing the data and trying to identify the similarities with the existing data and tries to find suitable solutions to existing and available problems. Studies of the scientists now a day's revealed that the predictions that were being generated by the machines with artificial intelligence are giving the more accurate and more correct results when compared with the other systems and other human beings[4]. It is true that, humans may fail or may commit some small marginal errors while calculating or predicting the astronomical data, but the machines may not commit such mistakes. 


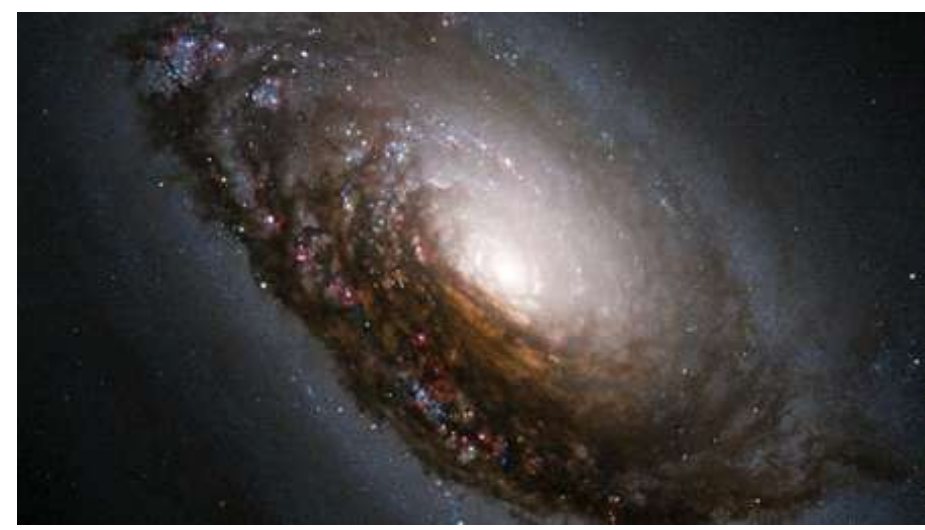

[Fig. 1] An Example for Astronomical Applications[5]

By using the artificial intelligence techniques and methods in these sorts of applications, the studies will give us the detailed results or the details about the universe and their functionalities or the way it is working. It is also provided that the details about the changes or alterations we can make to the existing system might also be available to the users who can use it for further research and more analysis.

\subsection{Construction}

The other important application areas of artificial intelligence were the construction sector. The most of the people in the society believe that the utilization of artificial intelligence in the current sector will be a great advantage for the sector as it was giving good benefits to the public. Many applications of artificial intelligence are being used today in the construction sector. The utilization of artificial intelligence could suggest the best design features of the buildings and the best safety measures for the construction of building with good design and best safety features. By providing the best designs of the buildings, the living lives of the people are becoming smoother and more comfortable. It will also be used in risk mitigation and reduction of human risks for the public. The safety measures will be very high very standard such that the living lives quality will be enhanced[6].

The utilization of artificial intelligence techniques, the engineers will get the good output and at the same time, they can predict the quality and standards of the constructions they are planning and the productivity will be more as per expected one. The more quality outcome or the output can be expected from the same system with less amount of time. The engineers can analyze the status of the work going on in the construction site and can make changes to the existing ones at regular intervals of time. 


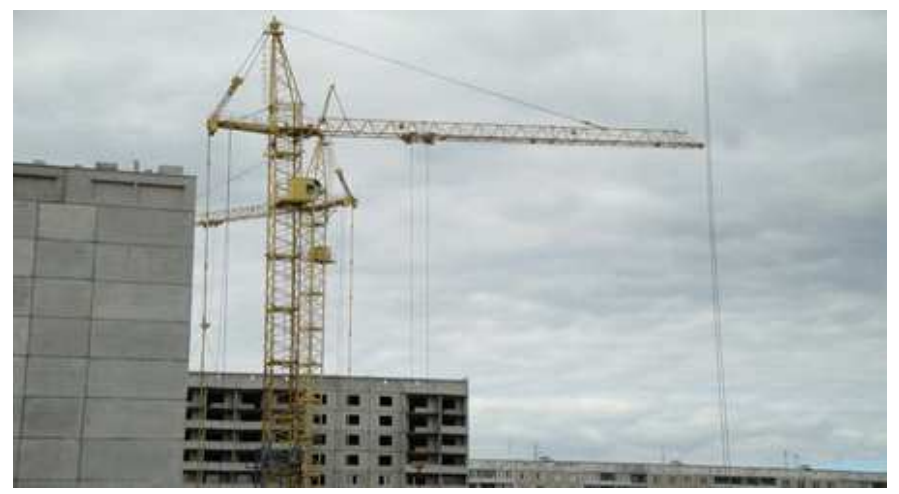

[Fig. 2] An Example for Construction Applications of $\mathrm{AI}[7]$

\subsection{Agriculture}

As far as concerned, for the developing countries like India, agriculture is the backbone of the country. Almost $80 \%$ of the people in the country are living with the agriculture and its related areas of livelihood. Hence, giving support to that area of work and other best suggestions to the farmers to get the good yields at correct times, suggest them to avoid cyclone problems, drought situations and suggest them to get good yields are the most important consideration one should have. The artificial intelligence will have a vital challenges and very good scope to work and implement the techniques of $\mathrm{AI}$ in this field. The utilization of these techniques in this sector may also some ground level problems. The other important area where the utilization was required $n$ agriculture sector was the IoT[8].

The utilization of these techniques will help in predicting the future of the crop, what sort of problems or the bacteria may attack the crop and try to find the best solution to avoid such circumstances. The other important consideration was to identify the rainfall in the area where the amount of rain may fall such that the farmers can plan for the appropriate crop such that they may not face any problem $f$ lack of rain water or ground water for the growth of the crops. It is also important to identify the amount of pesticides or the amount of urea required to be utilized for the crop based on the situation. These sort of some applications can be used for the AI techniques such that to guide the farmers in the correct direction. The farmers need to update with the technologies that were changing from time to time and observe the changes adopt them from time to time to get more benefits and other related yields for the same. 
The other applications of the AI are used in the agriculture field such that to get good results or the good yields for identifying the diseases that may attack the crops from time to time. Most of the diseases may depend on the area of the crop cultivation, the rainfall amount, rainfall season, the wind directions and the amount of daylight it is being coming to crops and the amount of fertilizers we are using for the crop to be increase of yield. But, it is always not possible to get good yield as it very important. Hence, guiding the farmers from time to time was the duty of the researchers such that o help the farmers to get good yields and that might increase the potential backbone of the country. The research always should concentrate on the factors that the farmers should get good results and good yields whenever farmers are cultivating with less expenditure and good yields.

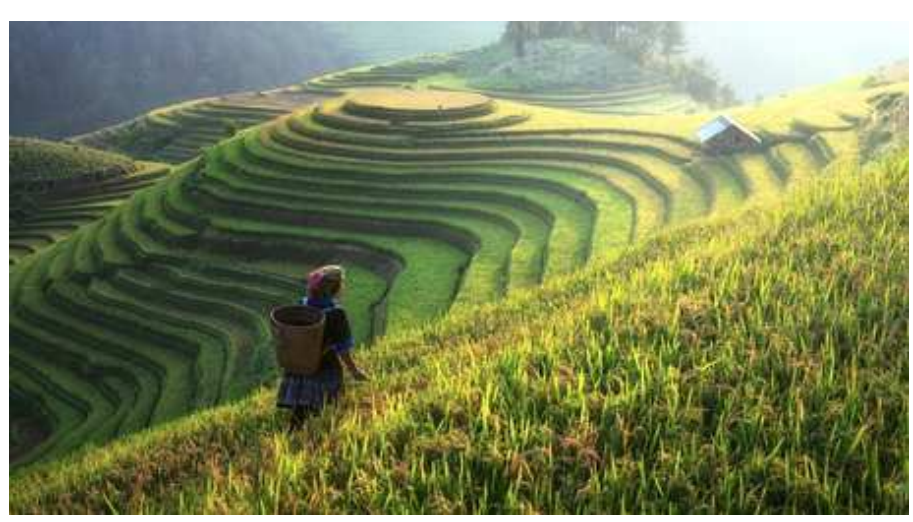

[Fig 3] An Agriculture Example Picture of Artificial Intelligence[7]

\subsection{Sports}

Now a day, the utilization of artificial intelligence and its techniques in the field of sports and games may change the entire worlds view of looking towards the sports and other latest trends in the sports. The total scenario was changed for the applications and utility of games had brought the dramatic changes. This trend of using AI techniques in sports had changed the world's perspective towards the games and brought the changes in the sports world. The utilization of these techniques might change the interests of the public towards the games and the sports people's perspective changed towards the games and the players for utilization of AI techniques[9]. 


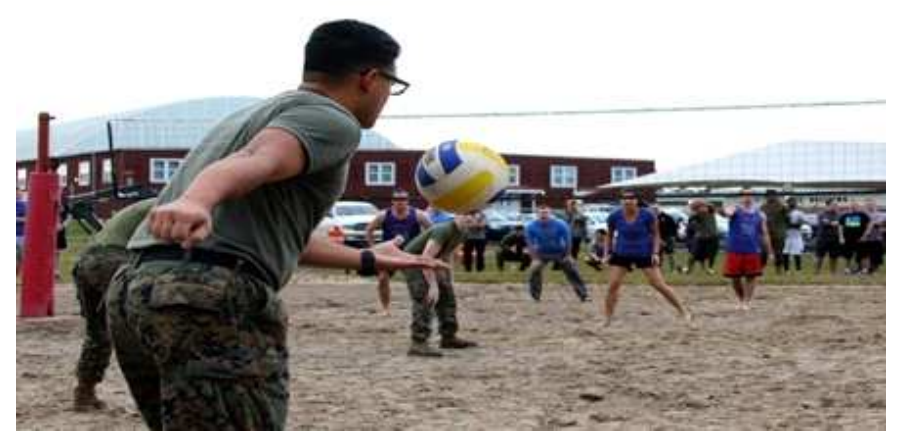

[Fig. 4] An Example of AI Utilization in Sports[7]

The practice of the sports persons had increases a lot due to the availability of sophisticated technology and other devices such that the players can increase their workout time such that they may get good results in the competitions in the national level or at the international level. The players and athletes can increase their workout time very high due to the availability of these sophisticated features.

\subsection{Entertainment}

In the recent days and years, the utilization of artificial intelligence techniques and other models for the better output from the entertainment and music applications. The entire format of the music and its related applications had changed the way we are using the music instruments and their applications. With the utilization of various AI techniques, the performance of the music instruments and other equipments was increased a lot. Now days, we can observe the music instruments and media communications with wireless communications and working smartly without the press of the human fingers or the human touch. In the earlier days, the touch of a human being is required for operating a music instrument, but now a day the instrument can be observed and played wirelessly with the help of these techniques and models.

The implementation of $\mathrm{AI}$ and its related applications and techniques were imposing in almost all applications and the utility of these applications are bringing a great change and great comfort in our day to day lives. 


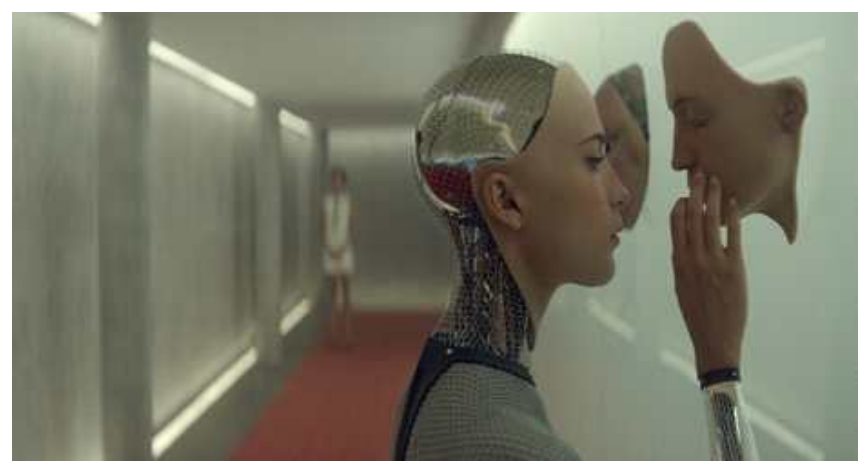

[Fig. 5] An Example of Entertainment Applications of AI[7]

\subsection{Education}

The other important application of artificial intelligence techniques was the education system. The implementation of good and advanced techniques in the education system was to upgrade the levels of thinking for the students and the application of these techniques to get good research results for the better of the existing technologies. The utilization of these techniques is making our lives and education system smarter and more advanced and with more flexibility. With the incorporation of these techniques and other features, the teaching learning blend of things is also changing a lot from the student point of view and the teacher point of view[10].

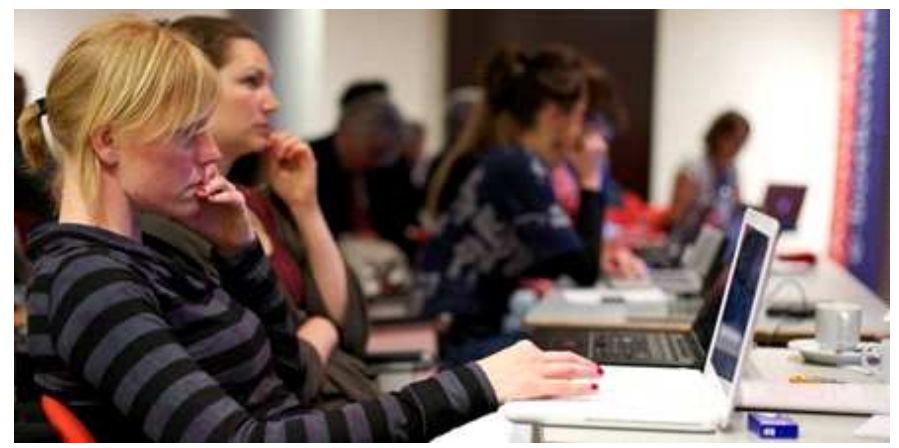

[Fig. 6] An Education and Teaching Example of $\mathrm{AI}[7]$

\subsection{Healthcare}

The utilization of artificial intelligence and its related techniques in the field of medical and its related applications and other healthcare related applications is increasing day to day. The improvement in the area of medical research and the utilization was more and more. Now a day, the related techniques are being utilized very more and other related applications. The 
identification of the diseases had become very easy and the proper treatment for the same disease can be given easily. The diseases can be identified easily and at the right time and also it is possible to identify the diseases that may be attacked to particular patients based on the symptoms with the person in advance. As a result of this, the preventive measures can be taken at the early stages such that the disease can be reduced or can be prevented.

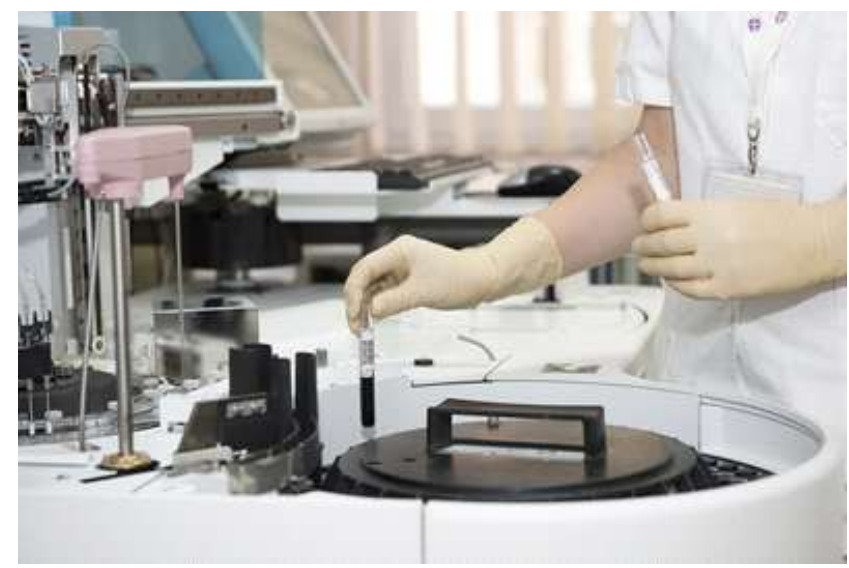

[Fig. 7] An Example of AI Techniques and Methods in Medical and Healthcare Applications[7]

The discovery of various drugs for the new diseases and the checking of the functioning of such diseases also can be verified with the help of these techniques. After the implementation of these techniques, the drug discovery and drug quality of functioning also became very easy.

\section{Conclusion}

In the current paper, the authors had given the brief details about the various techniques and other methods that were related to the artificial intelligence. The implementation of these techniques in various sectors and applications may give various type of benefits to the common people to the business people in the society. The life of the people in the society is becoming smarter and smarter by day to day as days are passing. The utilization of these techniques are increasing from day to day in the lives of the public and becoming more powerful by day to day. 


\section{References}

[1] Rupali Kamble, Deepali Shah, Applications of Artificial Intelligence in Human Life, International Journal of Research Granthaalayah, Vol.6, No.6, pp.178-188.

[2] E. Tyugu, Artificial intelligence in cyber defense, 3rd International Conference on Cyber Conflict (ICCC 2011), (2011), June 7-10; Tallinn, Estonia

[3] J. Helano, M. Nogueira, Mobile Intelligent Agents to Fight Cyber Intrusions, International Journal of Forensic Computer Science (IJoFCS), (2006), Vol.1, pp.28-32.

[4] Avneet Pannu, Artificial Intelligence and its Application in Different Areas, International Journal of Engineering and Innovative Technology, Vol.4, No.10, pp.79-84.

[5] James Vincent, A tutorial on AI could be the perfect tool for exploring the Universe, The Real World AI Issues, (2017)

[6] Rusul Abduljabbar, Hussein Dia, Sohani Liyanage and Saeed Asadi Bagloee, Applications of Artificial Intelligence in Transport: An Overview, Sustainability, (2019), Vol.11, No.1, pp.189-199.

[7] https://learntechx.com/blog/applications-of-artificial-intelligence-in-top-10-areas, Feb 10 (2019)

[8] X. B. Wang, G. Y. Yang, Y. C. Li, D. Liu, Review on the application of Artificial Intelligence in ntivirus Detection System, IEEE Conference on Cybernetics and Intelligent Systems, (2011), Sept 21-24; Chengdu, China

[9] A. S. Poonia, A. Bhardwaj, G. S. Dangayach, Cyber Crime: Practices and Policies for Its Prevention, The First International Conference on Interdisciplinary Research and Development, Special No. of the International Journal of the Computer, the Internet and Management, (2011), May 31-June 1; Thailand

[10] S. Gordon, R. Ford, On the definition and classification of cybercrime, Journal in Computer Virology, (2016), Vol.2, No.1, pp.13-20. 